Research Paper

\title{
Characterization of the Complete Mitochondrial Genome Sequence of Spirometra erinaceieuropaei (Cestoda: Diphyllobothriidae) from China
}

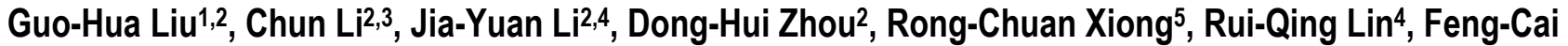 \\ Zou $^{6,2}$, Xing-Quan Zhu²,1,6 $\bowtie$
}

1. College of Veterinary Medicine, Hunan Agricultural University, Changsha, Hunan Province 410128, China;

2. State Key Laboratory of Veterinary Etiological Biology, Key Laboratory of Veterinary Parasitology of Gansu Province, Lanzhou Veterinary Research Institute, Chinese Academy of Agricultural Sciences, Lanzhou, Gansu Province 730046, China;

3. Sichuan Center for Animal Disease Control and Prevention, Chengdu, Sichuan Province 610041, China;

4. College of Veterinary Medicine, South China Agricultural University, Guangzhou, Guangdong Province 510642, China;

5. Chengdu Institute of Biology, Chinese Academy of Sciences, Chengdu 610041, China;

6. College of Animal Science and Technology, Yunnan Agricultural University, Kunming, Yunnan Province 650201, China.

$\square$ Corresponding author: Prof. Xing-Quan Zhu, State Key Laboratory of Veterinary Etiological Biology, Lanzhou Veterinary Research Institute, CAAS, Lanzhou, Gansu Province 730046, China. Phone: 86-931-8342837; Fax: 86-931-8340977; Email: xingquanzhu1@hotmail.com or Assoc Prof. Feng-Cai Zou, College of Animal Science and Technology, Yunnan Agricultural University, Kunming, Yunnan Province 650201, China. Phone: 86-871-5222291; Fax: 86-871-5222291; Email: zfc1207@vip.163.com.

(C) Ivyspring International Publisher. This is an open-access article distributed under the terms of the Creative Commons License (http://creativecommons.org/ licenses/by-nc-nd/3.0/). Reproduction is permitted for personal, noncommercial use, provided that the article is in whole, unmodified, and properly cited.

Received: 2012.01.15; Accepted: 2012.04.20; Published: 2012.04.27

\begin{abstract}
Sparganosis, caused by the plerocercoid larvae of members of the genus Spirometra, can cause significant public health problem and considerable economic losses. In the present study, the complete mitochondrial DNA (mtDNA) sequence of Spirometra erinaceieuropaei from China was determined, characterized and compared with that of $S$. erinaceieuropaei from Japan. The gene arrangement in the $\mathrm{mt}$ genome sequences of $\mathrm{S}$. erinaceieuropaei from China and Japan is identical. The identity of the $\mathrm{mt}$ genomes was $99.1 \%$ between $\mathrm{S}$. erinaceieuropaei from China and Japan, and the complete mtDNA sequence of $S$. erinaceieuropaei from China is slightly shorter (2 bp) than that from Japan. Phylogenetic analysis of S. erinaceieuropaei with other representative cestodes using two different computational algorithms [Bayesian inference (BI) and maximum likelihood (ML)] based on concatenated amino acid sequences of 12 protein-coding genes, revealed that $S$. erinaceieuropaei is closely related to Diphyllobothrium spp., supporting classification based on morphological features. The present study determined the complete mtDNA sequences of $S$. erinaceieuropaei from China that provides novel genetic markers for studying the population genetics and molecular epidemiology of S. erinaceieuropaei in humans and animals.
\end{abstract}

Key words: Spirometra erinaceieuropaei, sparganosis, mitochondrial genome, mitochondrial DNA, phylogenetic analyses.

\section{Introduction}

Cestodes (Cestoda, Platyhelminthes) are a highly diversified group of parasites, and the majority of cestodes can cause serious diseases in humans and animals globally, such as dipylidiasis, hydatidosis, cysticercosis, diphyllobothriasis and sparganosis [1]. Human sparganosis is a neglected parasitic disease caused by the plerocercoid larvae (spargana) of members of the genus Spirometra [2]. Spirometra erina- 
ceieuropaei and S. mansonoides are responsible for sparganosis in Asia and Americas, respectively [3].

Mitochondrial DNA (mtDNA) sequences have provided genetic markers for addressing questions in systematics and population genetics of Spirometra cestodes. A recent study showed that mtDNA cox1 sequences are useful genetic markers for the identification of species within Spirometra [4]. Employing mtDNA cox 1 gene sequences, a previous study demonstrated that S. erinaceieuropaei and S. proliferum are distinct species [5]. Although the single gene or single region of mtDNA have been considered as useful molecular markers, the complete mt genome sequences have significantly improved the power and resolution of phylogenetic analysis compared with single gene or single region of mtDNA [6-8]. Additionally, the complete $\mathrm{mt}$ genome sequences allow for more accurate resolution of taxonomic relationships even at deep levels than single gene or single region of mtDNA [9].

Metazoan mitochondrial $(\mathrm{mt})$ genomes contain 36-37 genes, including 12-13 protein-coding genes (PCGs), two ribosomal RNAs (rRNA) genes and 22 transfer RNAs (tRNA) genes necessary for translation of the proteins encoded by mt DNA (mtDNA) [10]. mtDNA sequences have been widely used as genetic markers not only for studies of population or ecological genetics, but also for phylogenetic and evolutionary analyses due to its maternal inheritance, rapid evolutionary rate, lack of recombination, and relatively conserved genome structures [11-15]. Although Cestoda is a large animal class, to date the complete $\mathrm{mt}$ genomes of only 19 species have been sequenced and deposited in GenBank. In particular, in the order Pseudophyllidea, the complete $\mathrm{mt}$ genomes of only three species have been reported, namely Diphyllobothrium latum and D. nihonkaiense [4], and S. erinaceieuropaei from Japan (GenBank accession No. NC_011037). The $\mathrm{mt}$ genome of S. erinaceieuropaei from a snake in Japan has been sequenced, but S. erinaceieuropaei isolated from different hosts and different geographic origins may have some differences because a previous study indicated that $\mathrm{mt}$ genomes for $N$. americanus from two endemic regions showed substantial sequence variation [16]. Moreover, the $\mathrm{mt}$ genome of S. erinaceieuropaei from Japan has not been characterized yet.

The objectives of the present study were: (i) to characterise the $\mathrm{mt}$ genome of $S$. erinaceieuropaei from China, (ii) to compare this mt genome with that of $S$. erinaceieuropaei from snake in Japan, (iii) to test the hypothesis that S. erinaceieuropaei from different hosts and different geographic origins have significant nucleotide sequence differences in their $\mathrm{mt}$ genomes, and (iv) to re-construct the phylogenetic relationships of the class Cestoda using mtDNA sequence data.

\section{Materials and Methods}

\section{Parasites and DNA extraction}

An adult cestode representing S. erinaceieuropaei was obtained from the small intestine of an infected farmed $\operatorname{dog}$ at a slaughterhouse in Zhanjiang, Guangdong province, China. The cestode was washed in physiological saline, identified preliminarily to species based on host preference, morphological characters and predilection sites [17], fixed in 70\% $(\mathrm{v} / \mathrm{v})$ ethanol and stored at $-20^{\circ} \mathrm{C}$ until use.

Total genomic DNA was isolated form this tapeworm using sodium dodecyl sulphate/proteinase $\mathrm{K}$ treatment, followed by spin-column purification (Wizard ${ }^{\circledR}$ SV Genomic DNA Purification System, Promega). The identity of this specimen was confirmed as $S$. erinaceieuropaei by polymerase chain reaction (PCR) amplification and subsequent sequencing of the ITS- 1 and ITS- 2 rDNA using primers reported previously [18].

\section{Amplification and sequencing of partial cox3, nad4, coxI and rrnS}

Initially, fragments of cox3, nad4 and rrnS genes were amplified individually from $S$. erinaceieuropaei (China isolate) by PCR with primers (Table 1) designed based on mtDNA sequences well-conserved in S. erinaceieuropaei (Japan isolate, GenBank accession No. NC_011037) (Table 2) by using Program Primer Primer 5.0 (PREMIER Biosoft International). Primers JB3 and JB4.5 [19] were utilized to amplify the partial cox1 gene of S. erinaceieuropaei (China isolate) (Table 1). PCR reactions $(25 \mathrm{~mL})$ were performed in $10 \mathrm{mM}$ Tris- $\mathrm{HCl}$ (pH 8.4), $50 \mathrm{mM} \mathrm{KCl}, 4 \mathrm{mM} \mathrm{MgCl} 2,200 \mathrm{mM}$ each of dNTP, 50 pmol of each primer and $2 \mathrm{U}$ Taq polymerase (Takara) in a thermocycler (Biometra) under the following conditions: after an initial denaturation at $94{ }^{\circ} \mathrm{C}$ for $5 \mathrm{~min}$, then 36 cycles at $94{ }^{\circ} \mathrm{C}$ for $30 \mathrm{~s}$ (denaturation), $55^{\circ} \mathrm{C}$ (for $\operatorname{cox} 1$ ) or $50^{\circ} \mathrm{C}$ (for $\operatorname{cox} 3$ ) or $54^{\circ} \mathrm{C}$ (for nad4 and $r r n S$ ) for $30 \mathrm{~s}$ (annealing), $72{ }^{\circ} \mathrm{C}$ for $30 \mathrm{~s}$ (extension), followed by $72{ }^{\circ} \mathrm{C}$ for $5 \mathrm{~min}$ (final extension). One microliter (5-10 ng) of genomic DNA was added to each PCR reaction. Each amplicon $(5 \mu \mathrm{L})$ was examined by $(1 \%)$ agarose gel electrophoresis. 
Table I. Sequences of primers used to amplify PCR fragments from Spirometra erinaceieuropaei.

\begin{tabular}{ll}
\hline Name of primer & Sequence $\left(5^{\prime}\right.$ to $\left.3^{\prime}\right)$ \\
\hline Short-PCR & \\
cox3-F & TGCATTTTGGTTATTTATTCTTAG \\
cox3-R & ACGATAGGCCCCGGCTGAAG \\
nad4-F & GGTTCCGTTATTTCCATTTCA \\
nad4-R & TACTACCCTCAAAAGACTCACCACG \\
JB3 & TTTTTGGGCATCCTGAGGTTTAT \\
JB4.5 & TAAAGAAAGAACATAATGAAAATG \\
rrnS-F & ATTGCGTAGTGAGGGGGATTA \\
rrnS-R & CTGGGGCTACCTTGTTACGACTTA \\
Long-PCR & \\
cox3-F & TGCATTTTGGTTATTTATTCTTAG \\
nad4-R & TACTACCCTCAAAAGACTCACCACG \\
SEND41F & CTGCGAGGTATTTGTGCTGTCTTCTTCA \\
SECO11R & CACAGGCTCACGCAACGAAACACGACTA \\
JB3 & TTTTTGGGCATCCTGAGGTTTAT \\
rrnS-R & CTGGGGCTACCTTGTTACGACTTA \\
SERNS1F & CCTGTAATGGTTTATGTTTTAGGACTTG \\
SECO31R & CAAAATGTCAATACCAAGTAACTAAAG \\
\hline
\end{tabular}

Table 2. Mitochondrial genome organization of Spirometra erinaceieuropaei from China and Japan.

\begin{tabular}{|c|c|c|c|c|c|}
\hline \multirow[t]{2}{*}{ Gene/region } & \multicolumn{2}{|c|}{ Positions and nt size (bp) } & \multicolumn{2}{|c|}{ Ini/Ter codon } & \multirow[t]{2}{*}{ Anticodons } \\
\hline & SEC & SEJ & SEC & SEJ & \\
\hline tRNA-Tyr (Y) & $1-68(68)$ & $1-68(68)$ & & & GTA \\
\hline Non-coding region (NC) & $69-272(204)$ & 69-272 (204) & & & \\
\hline tRNA-LeuCUN (L1) & $273-340(68)$ & $273-340(68)$ & & & TAG \\
\hline tRNA-SerUCN (S2) & $343-407$ (65) & $343-407$ (65) & & & TGA \\
\hline tRNA-LeuUUR (L2) & $412-476(65)$ & $412-476(65)$ & & & TAA \\
\hline tRNA-Arg (R) & $492-548(57)$ & $492-548(57)$ & & & ACG \\
\hline nad5 & $552-2117(1566)$ & $552-2120(1569)$ & ATG/TAA & ATG/TAA & \\
\hline Non-coding region (NR) & 2118-2291 (174) & 2121-2294 (174) & & & \\
\hline tRNA-Gly (G) & $2292-2368(67)$ & $2295-2361(67)$ & & & TCC \\
\hline $\cos 3$ & $2362-3004(643)$ & $2365-3007(643)$ & GTG/T & GTG/T & \\
\hline tRNA-His (H) & $3005-3073(69)$ & 3008-3076 (69) & & & GTG \\
\hline$c y t b$ & $3077-4186$ (1110) & $3080-4189$ (1110) & ATG/TAA & ATG/TAA & \\
\hline $\operatorname{nad} 4 \mathrm{~L}$ & $4191-4451(261)$ & $4194-4454(261)$ & ATG/TAG & ATG/TAG & \\
\hline nad4 & $4412-5665$ (1254) & $4415-5668$ (1254) & ATG/TAG & ATG/TAG & \\
\hline tRNA-Gln (Q) & $5666-5729(64)$ & $5669-5732(64)$ & & & TTG \\
\hline tRNA-Phe (F) & $5726-5789(64)$ & $5729-5792(64)$ & & & GAA \\
\hline tRNA-Met (M) & $5786-5853(68)$ & $5789-5856(68)$ & & & CAT \\
\hline atp6 & $5857-6372(516)$ & $5860-6375(516)$ & ATG/TAA & ATG/TAA & \\
\hline $\operatorname{nad} 2$ & $6380-7252(873)$ & 6383-7255 (873) & ATG/TAG & ATG/TAG & \\
\hline tRNA-Val (V) & $7263-7327$ (65) & $7266-7330(65)$ & & & TAC \\
\hline tRNA-Ala (A) & $7345-7405$ (61) & 7348-7408 (61) & & & TGC \\
\hline tRNA-Asp (D) & 7411-7474 (64) & 7414-7477 (64) & & & GTC \\
\hline $\operatorname{nad} 1$ & $7475-8365$ (891) & 7478-8368 (891) & ATG/TAA & ATG/TAA & \\
\hline tRNA-Asn (N) & $8371-8436(66)$ & $8374-8439$ (66) & & & GTT \\
\hline tRNA-Pro (P) & $8443-8508$ (66) & $8446-8510(65)$ & & & TGG \\
\hline tRNA-Ile (I) & $8514-8577$ (64) & $8516-8579$ (64) & & & GAT \\
\hline
\end{tabular}




\begin{tabular}{|c|c|c|c|c|c|}
\hline \multirow[t]{2}{*}{ Gene/region } & \multicolumn{2}{|c|}{ Positions and nt size (bp) } & \multicolumn{2}{|c|}{ Ini/Ter codon } & \multirow[t]{2}{*}{ Anticodons } \\
\hline & $\overline{\text { SEC }}$ & SEJ & SEC & SEJ & \\
\hline tRNA-Lys (K) & $8584-8646(63)$ & $8586-8648(63)$ & & & CTT \\
\hline nad3 & $8650-8995(346)$ & $8652-8997(346)$ & ATG/T & ATG/T & \\
\hline tRNA-SerAGN (S1) & 8996-9054 (59) & $8998-9056$ (59) & & & GCT \\
\hline tRNA-Trp (W) & $9057-9122(66)$ & $9059-9124(66)$ & & & TCA \\
\hline $\operatorname{cox} 1$ & 9130-10695 (1566) & 9132-10697 (1566) & ATG/TAG & ATG/TAG & \\
\hline tRNA-Thr (T) & $10686-10755(70)$ & $10688-10757(70)$ & & & TGT \\
\hline$r r n \mathrm{~L}$ & $10756-11728$ (973) & $10758-11730$ (973) & & & \\
\hline tRNA-Cys (C) & $11729-11793(65)$ & $11731-11795$ (65) & & & GCA \\
\hline$r r n S$ & $11794-12523(730)$ & $11796-12525(730)$ & & & \\
\hline $\cos 2$ & $12524-13093(570)$ & $12526-13095(570)$ & ATG/TAA & ATG/TAA & \\
\hline tRNA-Glu (E) & $13099-13163$ (65) & $13101-13165(65)$ & & & TTC \\
\hline nad6 & $13168-13635$ (468) & $13170-13637$ (468) & ATG/TAA & ATG/TAA & \\
\hline
\end{tabular}

SEC: S. erinaceieuropaei (China isolate), SEJ: S. erinaceieuropaei (Japan isolate).

Ini/Ter codons: initiation and termination codons.

\section{Long-PCR amplification and sequencing}

The obtained partial cox $1, \operatorname{cox} 3$, nad4 and rrnS sequences were compared with the corresponding sequences of $S$. erinaceieuropaei $\mathrm{mt}$ genome (Japan isolate, NC_011037) using the program Clustal X 1.83 [20], and then primers (Table 1) were designed in the conserved regions to amplify the complete $\mathrm{mt}$ genome of S. erinaceieuropaei (China isolate). Long-PCR reactions $(25 \mu \mathrm{l})$ were performed in $2 \mathrm{mM} \mathrm{MgCl} 2,0.2 \mathrm{mM}$ each of dNTPs, $2.5 \mu 1$ 10× rTaq buffer, $2.5 \mu \mathrm{M}$ of each primer, $1.25 \mathrm{U}$ rTaq polymerase (Takara), and $1 \mu \mathrm{l}$ of DNA sample in a thermocycler (Biometra) under the following conditions: $92^{\circ} \mathrm{C}$ for $2 \mathrm{~min}$ (initial denaturation), then $92{ }^{\circ} \mathrm{C}$ for $10 \mathrm{~s}$ (denaturation), $50{ }^{\circ} \mathrm{C}$ or 55 ${ }^{\circ} \mathrm{C}$ for $30 \mathrm{~s}$ (annealing), and $60^{\circ} \mathrm{C}$ for $10 \mathrm{~min}$ (extension) for 10 cycles, followed by $92^{\circ} \mathrm{C}$ for $10 \mathrm{~s}, 50^{\circ} \mathrm{C}$ or $55^{\circ} \mathrm{C}$ for $30 \mathrm{~s}$, and $60^{\circ} \mathrm{C}$ for $10 \mathrm{~min}$ for 20 cycles, with a cycle elongation of $10 \mathrm{~s}$ for each cycle and a final extension at $60^{\circ} \mathrm{C}$ for $10 \mathrm{~min}$. Samples not containing DNA (no-DNA controls) were included in each amplification run, and in neither case were amplicons detected in the no-DNA controls (data not shown). Each amplicon $(5 \mu \mathrm{L})$ was examined by agarose $(1 \%)$ gel electrophoresis, stained with ethidium bromide and photographed using a gel documentation system (UVItec). PCR products were sent to Sangon Company (Shanghai, China) for sequencing from both directions using a primer walking strategy.

\section{Sequence analyses}

Sequences were assembled manually and aligned against the complete $\mathrm{mt}$ genome sequence of
S. erinaceieuropaei (Japan isolate) using the computer program Clustal X 1.83 to identify gene boundaries. Sequence differences between the S. erinaceieuropaei China and Japan isolates were calculated by pairwise comparisons using the formula $D=1-(M / L)$, where $M$ is the number of alignment positions at which the two sequences have a base in common, and $L$ is the total number of alignment positions over which the two sequences are compared [21]. Gene annotation, genome organization, translation initiation, translation termination codons and the boundaries between PCGs of the $\mathrm{mt}$ genome sequence of $S$. erinaceieuropaei (China isolate) were identified based on comparison with the $\mathrm{mt}$ genome sequence of $S$. erinaceieuropaei (Japan isolate) reported previously. The amino acid sequences deduced from open-reading frames were aligned with those of S. erinaceieuropaei (Japan isolate) by using Clustal $X 1.83$. Based on pairwise comparison, amino acid identity (\%) was calculated for homologous genes. For analyzing ribosomal RNA genes, putative secondary structures of 22 tRNA genes were identified using tRNAscan-SE [22], or by recognizing potential secondary structures and anticodon sequences by eye by comparison with that of $S$. erinaceieuropaei (Japan isolate).

\section{Sliding window analysis of nucleotide variation}

To detect variable nucleotide sites, pairwise alignments of the complete genomes including tRNA and all intergenic spacers were performed using the program Clustal X 1.83. The complete alignment of nucleotides of the 4 Pseudophyllidea cestode mtDNAs was used to effect sliding window analyses 
using DnaSP v5 [23]. A sliding window of $300 \mathrm{bp}$ and steps of $10 \mathrm{bp}$ were used to estimate nucleotide diversity $\mathrm{Pi}$ (п) for the complete alignment. Nucleotide diversity for the complete alignment was plotted against mid-point positions of each window, and gene boundaries were indicated.

\section{Phylogenetic analyses}

Phylogenetic relationships among 19 cestode species were reconstructed using Ascaris suum (GenBank accession No. HQ704901), an ascaridoid nematode, as the outgroup, based on amino acid sequences of 12 PCGs. Each gene was translated into amino acid sequence using the invertebrate mitochondrial genetic code in MEGA 5.0 [24], and aligned on the basis of its amino acid sequence, using default settings, and ambiguously aligned regions were excluded using the Gblocks online server [25] (http://molevol.cmima. csic.es/castresana/Gblocks_server.html) using the options for a less stringent selection. The final amino acid sequences of the 12 PCGs were then concatenated into single alignments for phylogenetic analyses.

Two inference methods, namely, Bayesian inference (BI) and maximum likelihood (ML) were used for phylogenetic re-constructions. BI analyses were conducted with four independent Markov chains run for 1,000,000 metropolis-coupled MCMC generations, sampling a tree every 100 generations in MrBayes 3.1.1 [26]. The first 2,500 trees were omitted as burn-in and the remaining trees were used to calculate Bayesian posterior probabilities (PP). ML analyses were performed using PhyML 3.0 [27], and the $\mathrm{JTT}+\mathrm{G}+\mathrm{F}(\mathrm{lnL}=-50057.3646$, Gamma distribution parameter $a=0.5034$ ) model with its parameter for the concatenated dataset was determined for the ML analysis using MEGA 5.0 based on the Akaike information criterion (AIC). Bootstrap support for ML trees was calculated using 100 bootstrap replicates. Phylograms were drawn using the Tree View program version 1.65 [28].

\section{Results and Discussion}

\section{Genome organization and structure}

The complete $\mathrm{mt}$ genome of $S$. erinaceieuropaei (China isolate) was amplified in four overlapping long fragments between cox 3 and nad4 (approximately $3.1 \mathrm{~kb}$ ), between nad4 and cox1 (approximately $4.6 \mathrm{~kb}$ ), between cox 1 and rrnS (approximately $2.7 \mathrm{~kb}$ ) and between $\mathrm{rrnS}$ and cox3 (approximately $3.5 \mathrm{~kb}$ ). The complete $\mathrm{mt}$ genome sequence of $S$. erinaceieuropaei (China isolate) was 13,641 bp in size (Fig. 1), and it has been deposited in the GenBank under the accession number JQ267473. The S. erinaceieuropaei (China iso- late) mt genome sequence $(13,641 \mathrm{bp})$ was $2 \mathrm{bp}$ shorter than that of $S$. erinaceieuropaei (Japan isolate). The slight discrepancy of nucleotide size is likely due to difference in their hosts, developmental stages or geographical locations. The S. erinaceieuropaei China specimen (adult) was collected from a dog, while the S. erinaceieuropaei Japan isolate (larva) was collected from a snake. The $\mathrm{mt}$ genome of $S$. erinaceieuropaei (China isolate) contains 12 PCGs (cox1-3, nad1-6, nad $4 \mathrm{~L}$, atp6 and cytb), 22 transfer RNA genes, two ribosomal RNA genes and two non-coding regions (Table 2). All genes are transcribed in the same direction as other cestode species sequenced to date. These results indicate that the organization of $\mathrm{mt}$ genomes among sequenced cestodes is conserved, which may provide mtDNA evidence for the validity of the Cestoda as a unique animal class.

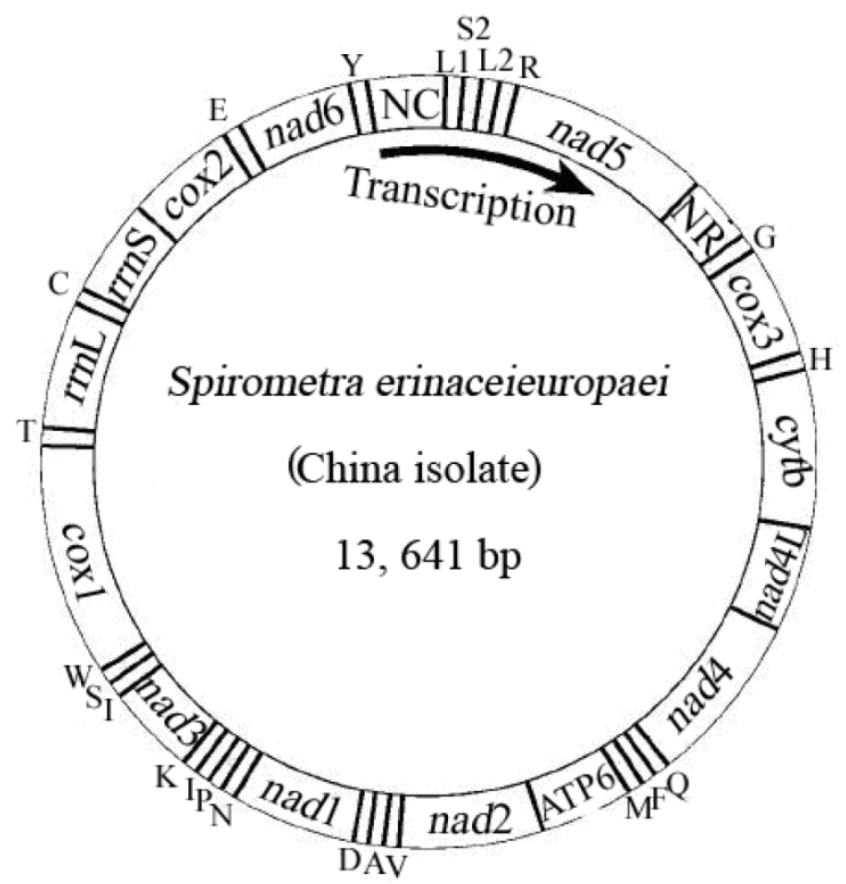

Fig. I Arrangement of the mitochondrial genome of Spirometra erinaceieuropaei (China isolate). Gene scaling is only approximate. All genes are coded by the same DNA strand and are transcribed clockwise. All genes have standard nomenclature except for the 22 tRNA genes, which are designated by the one-letter code for the corresponding amino acid, with numerals differentiating each of the two leucine- and serine-specifying tRNA (LI and L2 for codon families CUN and UUR, respectively; $S I$ and $S 2$ for codon families $A G N$ and UCN, respectively). "NR" refers to a small noncoding region. "NC" refers to a large noncoding region. 
The $\mathrm{mt}$ genome sequence of $S$. erinaceieuropaei (China isolate) has high T content and low $\mathrm{C}$ content ( $\mathrm{T}=45.9 \%, \mathrm{~A}=20.4 \%, \mathrm{G}=22.6 \%, \mathrm{C}=11.1 \%)$, and its $\mathrm{A}+\mathrm{T}$ content was $66.3 \%$, in accordance with $\mathrm{mt}$ genomes of other Pseudophyllidea species sequenced to date [4]. The identity of the nucleotide sequences between $\mathrm{mt}$ genomes of $S$. erinaceieuropaei (China isolate) and $S$. erinaceieuropaei (Japan isolate) is $99.1 \%$. Interestingly, the $\mathrm{mt}$ genomes of S. erinaceieuropaei (China and Japan isolates) have 20 intergenic regions, excluding the non-coding regions (Table 2). Even more noteworthy, the sizes and locations of these intergenic regions of both mt genomes were identical (Table 2). Furthermore, previous studies have shown that the intergenic regions of $\mathrm{mt}$ genome of metazoans contain signals for transcription initiation and replication [29].

\section{Protein-coding genes, transfer RNA genes, ribosomal RNA genes and non-coding regions}

The boundaries between PCGs of $\mathrm{mt}$ genome of S. erinaceieuropaei (China isolate) were determined by aligning their sequences and by identifying translation initiation and termination codons with comparison to those of S. erinaceieuropaei (Japan isolate). The longest genes are cox 1 and nad5 genes (Table 2).

A total of 3356 amino acids are encoded in the $\mathrm{mt}$ genome of S. erinaceieuropaei (China isolate). All PCGs use ATG or GTG as their initiation codon, which encodes methionine and valine that is consistent with those of S. erinaceieuropaei (Japan isolate). Although ATG is the most common initiation codon, unusual initiation codons such as GTG and GTT were also reported for some cestodes [30, 31]. All genes have complete termination codon, except for cox3 and nad3 genes which uses incomplete termination codon $(T)$ as termination codon. 6 genes (cox2, nad1, nad6, nad5, cytb and atp6) use TAA and 4 genes (cox1, nad2, nad4L and nad4) use TAG as termination codon. Incomplete termination codons ( $\mathrm{T}$ or TA) as termination codon are also present in other cestodes [4, 32].

The sizes of 22 tRNA genes identified in the $\mathrm{mt}$ genome of $S$. erinaceieuropaei (China isolate) ranged from 57 to 69 nucleotides (nt) with differences in stem and loop sizes of dihydrouridine (D) and TCC arms. Their putative secondary structures (not shown) are similar to those of other Pseudophyllidea cestodes [4], indicating their similar functions.

The $r r n \mathrm{~L}$ and $r r n S$ genes of S. erinaceieuropaei (China isolate) were identified by comparison with that of S. erinaceieuropaei (Japan isolate). The $r r n \mathrm{~L}$ is located between tRNA-Thr and tRNA-Cys, and $r r n S$ is located between tRNA-Cys and cox2. The sizes of the $r r n \mathrm{~L}$ and $r r n S$ genes are $973 \mathrm{bp}$ and $730 \mathrm{bp}$ both for $S$. erinaceieuropaei (China isolate) and S. erinaceieuropaei
(Japan isolate), respectively (Table 2). Sequence identity in the $r r n \mathrm{~L}$ and $r r n S$ genes are $98.6 \%$ and $99.3 \%$ between S. erinaceieuropaei (China isolate) and S. erinaceieuropaei (Japan isolate), respectively.

mtDNA sequences of most cestodes contain two non-coding regions with significant size difference. The large non-coding region (NC) in the $\mathrm{mt}$ genome of the S. erinaceieuropaei (China isolate) is located between the tRNA-Tyr and tRNA-LeuCUN, its size is 204 $\mathrm{bp}$, and its $\mathrm{A}+\mathrm{T}$ content is $71.6 \%$, whereas the small non-coding region (NR) in the $\mathrm{mt}$ genome of the $S$. erinaceieuropaei (China isolate) is located between the nad5 and tRNA-Gly, its length is $174 \mathrm{bp}$, and its A+T content is $63.8 \%$, which are identical to those of $S$. erinaceieuropaei (Japan isolate).

Moreover, the non-coding regions in S. erinaceieuropaei (both China and Japan isolates) contain continuous A or T base, similar to those of some Taenia species [13]. The non-coding regions are known to be related to the origin of replication in both vertebrates and invertebrates [10].

\section{Levels of variability in mtDNAs among Pseu- dophyllidea cestodes}

Sliding window analysis of the complete nucleotide alignment of 4 available Pseudophyllidea cestode mtDNAs provided an indication of nucleotide diversity $\mathrm{Pi}$ (п) within and between $\mathrm{mt}$ genes (Fig. 2). In the curve, the nucleotide variation within and between $\mathrm{mt}$ genes among the aligned Pseudophyllidea cestode mt genomes was intuitively displayed for any given window of $300 \mathrm{bp}$ and steps of $10 \mathrm{bp}$, with the $\Pi$ ranging from 0.055 to 0.297 . Coupled with computation of the number of variable positions per unit length of gene, the sliding window indicated that the PCGs with high sequence variability included nad6 (0.372), nad5 (0.37), nad2 (0.331), atp6 (0.316), nad3 (0.31) and nad4 (0.3), while the genes with low sequence variability included $\operatorname{cox} 3(0.299)$, cytb (0.263), nad4L (0.257), nad1 (0.251), cox1 (0.21) and cox 2 (0.195). In this study, the cox 2 and cox 1 genes are the most conserved PCGs, and nad6 and nad5 are the least conserved PCGs. Given the lowest and the highest sequence variability, cox2 (0.195) and nad6 (0.372) can be considered as the best markers in future studies of phylogenetics, population genetics and diagnostics of the Pseudophyllidea cestodes. Obviously, these genes are also more variable than the cox 1 gene in the present study ( 0.21 for $\operatorname{cox} 1)$, not consistent with that of previous studies $[33,34]$. Currently, mt genes such as cox 1 and cytb have been used as targets for PCR-based approaches for the identification and diagnosis of cestodes $[35,36]$. These results suggest that cox 1 gene may not be the most informative or most suitable for 
all cestode taxa although it has commonly been optioned as molecular markers. Our resultes indicated that sliding window analyses could define genetic markers for population genetics and systematics studies of cestodes.

\section{Nucleotide variation in mtDNA sequences between $S$. erinaceieuropaei from China and Japan}

Sliding window analysis of the nucleotide alignment of $S$. erinaceieuropaei mtDNA from China and Japan provided an indication of nucleotide diversity (п) within or between $\mathrm{mt}$ genes (Fig. 3). The plot readily shows the high degree of nucleotide variation within or between genes amongst the aligned $S$. erinaceieuropaei genomes for any given window of 300 bp (п) ranges from 0 to 0.043). Sliding window analysis indicates that genes with high sequence variability included cytb (0.023), nad4 (0.022) and cox1 (0.012). Genes with no sequence variability include nad1, cox2 and nad6. Based on these results, it seemed that nad1 and cox 2 are the most conserved protein-coding genes, and cytb and nad4 show high sequence variability between S. erinaceieuropaei from China and Japan. These results indicated that cytb and nad4 sequences provide alternative new genetic markers for population genetic studies of S. erinaceieuropaei from different hosts and different geographic origins.

\section{Phylogenetic analyses}

Phylogenetic analyses of cestodes using two methods (BI and ML) yielded identical tree topologies based on concatenated amino acid sequences of 12 protein genes, all revealed distinct groups with high statistical support, and demonstrated that S. erinaceieuropaei is closely related to Diphyllobothrium spp. (Fig. 4), consistent with that of a previous study using cox 1 sequences [37], supporting classification based on morphological features [38]. In this tree, Cyclophyllidea and Pseudophyllidea form monophyletic groups. Within the Cyclophyllidea clade, Hymenolepididae and Taeniidae form monophyletic groups. Within the Cyclophyllidea clade, Echinococcus and Taenia are sister genera.

In conclusion, the present study determined and characterized the complete mtDNA sequence of $S$. erinaceieuropaei from China, which is slightly shorter (2 $\mathrm{bp}$ ) than that of $S$. erinaceieuropaei from Japan, and both $\mathrm{mt}$ genomes differed by $0.9 \%$ only, refuted our hypothesis. These mtDNA sequences should provide novel genetic data for addressing further questions in systematics and population genetics of these and other related cestodes of socio-economic significance.

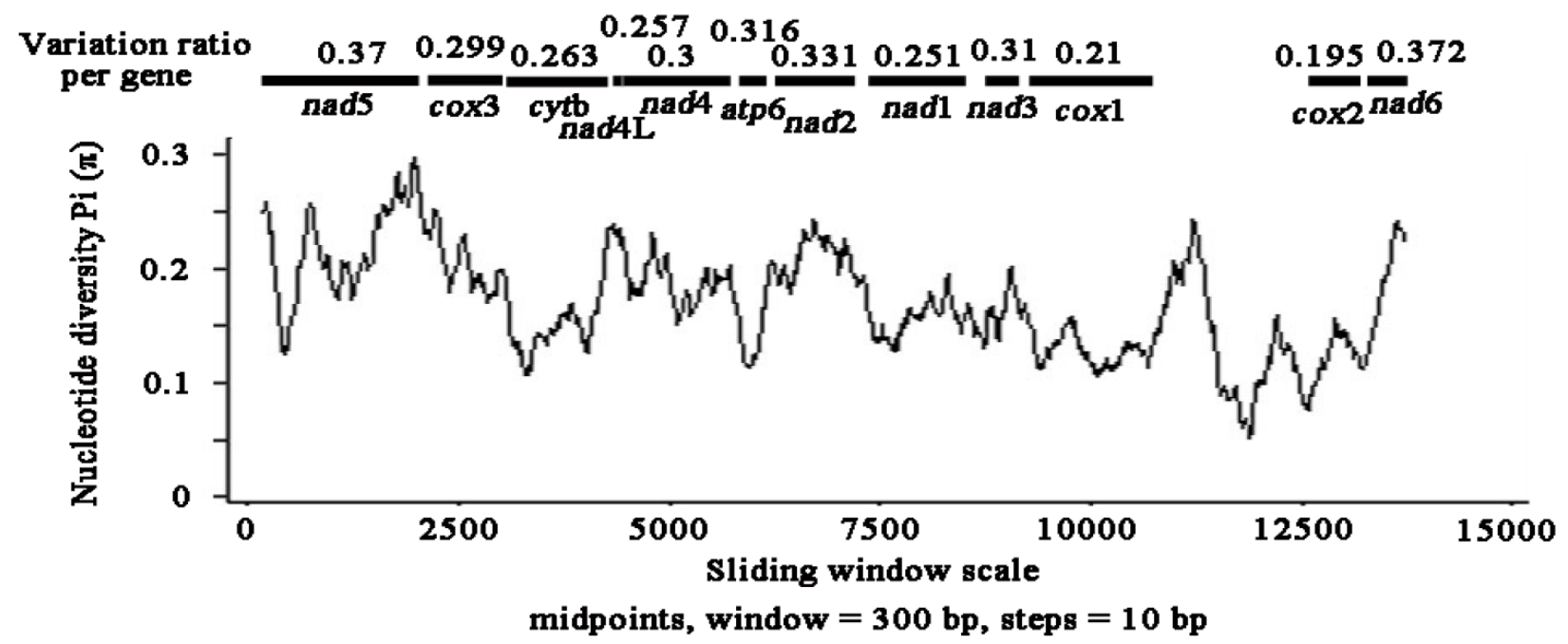

Fig. 2 Sliding window analysis of the alignment of complete mtDNAs of Pseudophyllidea cestodes. The black line shows the value of nucleotide diversity $\mathrm{Pi}(\pi)$ in a sliding window analysis of window size $300 \mathrm{bp}$ with step size 10 , and the value is inserted at its mid-point. Gene boundaries are indicated with a variation ratio per gene. 


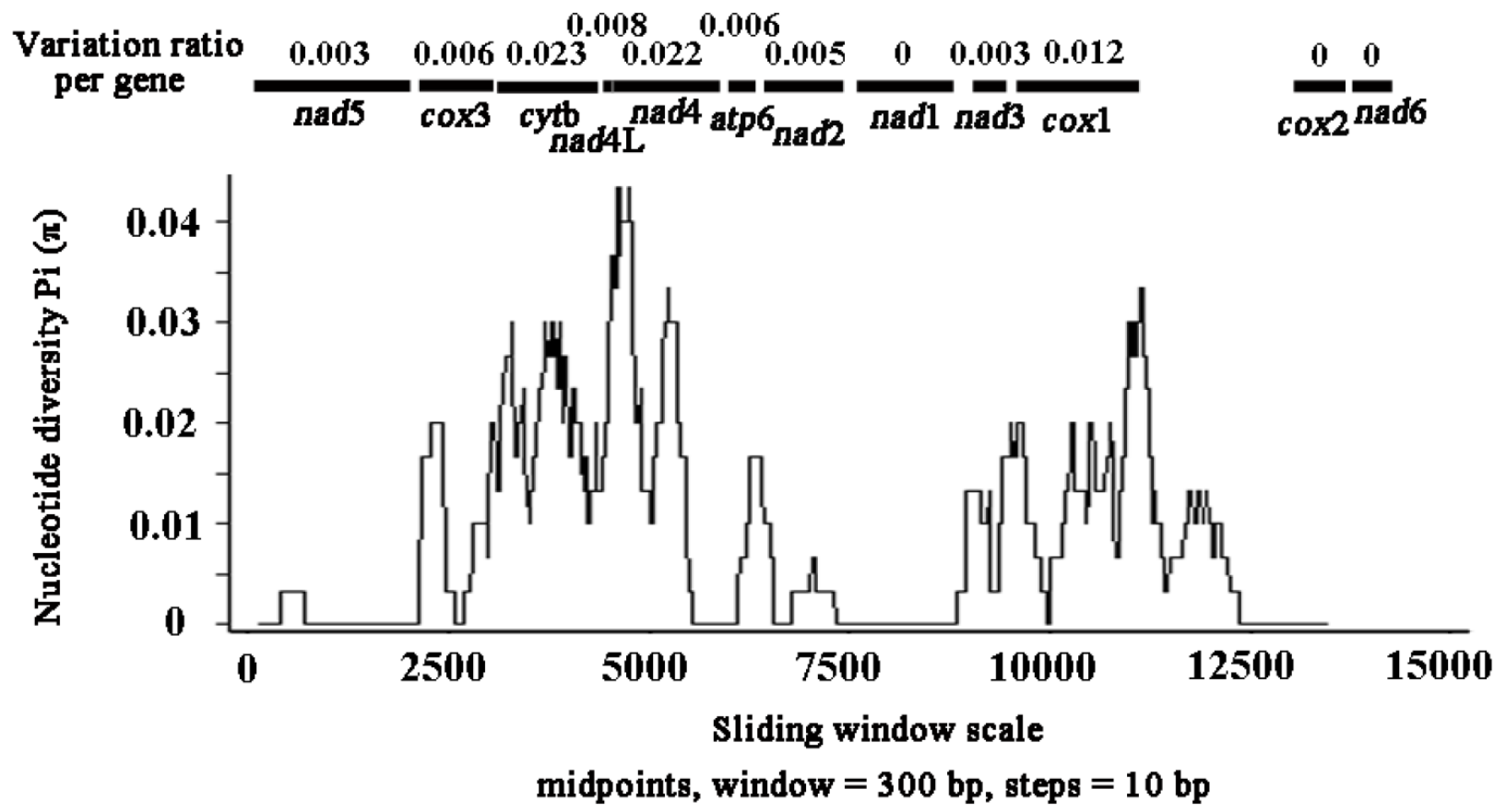

Fig. 3 Sliding window analysis of the alignment of complete mtDNAs of Spirometra erinaceieuropaei from China and Japan. The black line shows the value of nucleotide diversity $\mathrm{Pi}(\pi)$ in a sliding window analysis of window size $300 \mathrm{bp}$ with step size 10 , and the value is inserted at its mid-point. Gene boundaries are indicated with a variation ratio per gene.

$\mathrm{BI} / \mathrm{ML}$

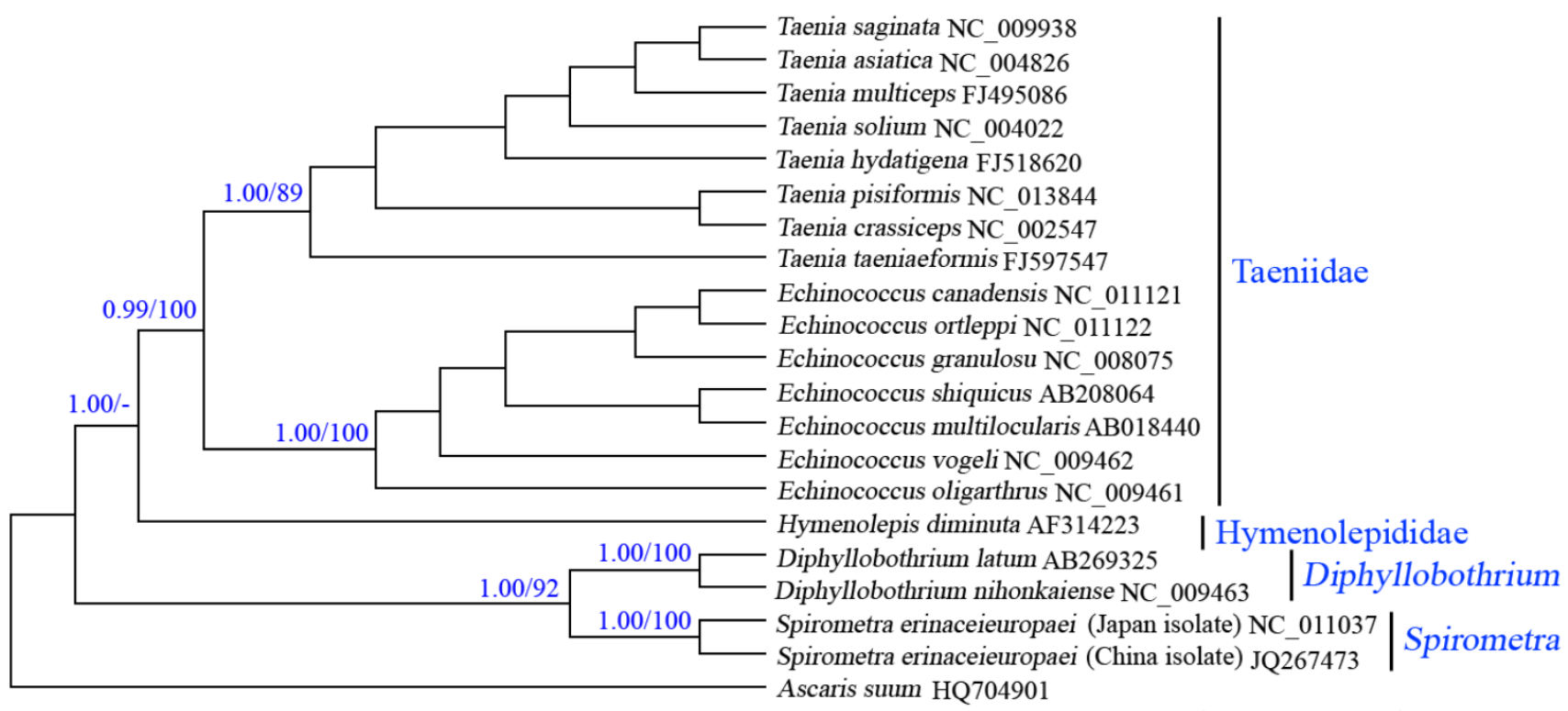

Fig. 4 Inferred phylogenetic relationships among cestodes based on amino acid sequences of I 2 mitochondrial protein-coding genes using Bayesian inference (Bayes) and maximum likelihood (ML) analysis, using Ascaruis suum (GenBank accession number $\mathrm{HQ70490I)}$ as the outgroup. The numbers along branches indicate posterior probability (PP) and bootstrap probability (BP) values resulting from different analyses in the order of Bayes/ML. Values lower than 70 are given as "-". 


\section{Acknowledgements}

This work was supported, in part, by the Program for Outstanding Scientists in Agricultural Research, the Open Funds of the State Key Laboratory of Veterinary Etiological Biology, Lanzhou Veterinary Research Institute, Chinese Academy of Agricultural Sciences (Grant Nos. SKLVEB2011KFKT011, SKLVEB2010KFKT009, SKLVEB2009KFKT008, SKLVEB2011KFKT010 and SKLVEB2011KFKT004) and the Yunnan Provincial Program for Introducing High-level Scientists (Grant No. 2009CI125).

\section{Competing Interests}

The authors have declared that no competing interest exists.

\section{References}

1. Spakulová M, Orosová M, Mackiewicz JS. Cytogenetics and chromosomes of tapeworms (Platyhelminthes, Cestoda). Adv Parasitol. 2011; 74: 177-230.

2. Li MW, Song HQ, Li C, Lin HY, Xie WT, Lin RQ, Zhu XQ. Sparganosis in mainland China. Int J Infect Dis. 2011; 15: e154-156.

3. Anantaphruti MT, Nawa Y, Vanvanitchai Y. Human sparganosis in Thailand: an overview. Acta Trop. 2011; 118: 171-176.

4. Nakao M, Abmed D, Yamasaki H, Ito A. Mitochondrial genomes of the human broad tapeworms Diphyllobothrium latum and Diphyllobothrium nihonkaiense (Cestoda: Diphyllobothriidae). Parasitol Res. 2007; 101: 233-236.

5. Miyadera H, Kokaze A, Kuramochi T, Kita K, Machinami R, Noya O, Alarcón de Noya B, Okamoto M, Kojima S. Phylogenetic identification of Sparganum proliferum as a pseudophyllidean cestode by the sequence analyses on mitochondrial COI and nuclear sdhB genes. Parasitol Int. 2001; 50: 93-104.

6. Santamaria M, Lanave C, Vicario S, Saccone C. Variability of the mitochondrial genome in mammals at the inter-species/intra-species boundary. Biol Chem. 2007; 388: 943-946.

7. Zardoya R, Meyer A. Phylogenetic performance of mitochondrial protein-coding genes in resolving relationships among vertebrates. Mol Biol Evol. 1996; 13: 933-942.

8. Douglas KC, Halbert ND, Kolenda C, Childers C, Hunter DL, Derr JN. Complete mitochondrial DNA sequence analysis of Bison bison and bison-cattle hybrids: function and phylogeny. Mitochondrion. 2011; 11: 166-175.

9. Gissi C, Iannelli F, Pesole G. Evolution of the mitochondrial genome of Metazoa as exemplified by comparison of congeneric species. Heredity. 2008; 101: 301-120.

10. Boore JL. Animal mitochondrial genomes. Nucleic Acids Res.1999; 27: $1767-1780$

11. Li MW, Lin RQ, Song HQ, Wu XY, Zhu XQ. The complete mitochondrial genomes for three Toxocara species of human and animal health significance. BMC Genomics. 2008; 9: 224.

12. Lin RQ, Qiu LL, Liu GH, Wu XY, Weng YB, Xie WQ, Hou J, Pan H, Yuan ZG, Zou FC, Hu M, Zhu XQ. Characterization of the complete mitochondrial genomes of five Eimeria species from domestic chickens. Gene. 2011; 480: 28-33.

13. Liu GH, Lin RQ, Li MW, Liu W, Liu Y, Yuan ZG, Song HQ, Zhao $\mathrm{GH}$, Zhang KX, Zhu XQ. The complete mitochondrial genomes of three cestode species of Taenia infecting animals and humans. Mol Biol Rep. 2011; 38: 2249-2256.

14. Li H, Gao J, Liu H, Liu H, Liang A, Zhou X, Cai W. The architecture and complete sequence of mitochondrial genome of an assassin bug Agriosphodrus dohrni (Hemiptera: Reduviidae). Int J Biol Sci. 2011; 7: 792-804.
15. Li H, Liu H, Cao L, Shi A, Yang H, Cai W. The complete mitochondrial genome of the damsel bug Alloeorhynchus bakeri (Hemiptera: Nabidae). Int J Biol Sci. 2012; 8: 93-107.

16. Hu M, Chilton NB, Abs El-Osta YG, Gasser RB. Comparative analysis of mitochondrial genome data for Necator americanus from two endemic regions reveals substantial genetic variation. Int J Parasitol. 2003; 33: 955-963.

17. Schmidt GD. Handbook of tapeworm identification. Boca Raton, FL: CRC Press. 1986.

18. Dai RS, Liu GH, Song HQ, Lin RQ, Yuan ZG, Li MW, Huang SY, Liu $\mathrm{W}$, Zhu XQ. Sequence variability in two mitochondrial DNA regions and internal transcribed spacer among three cestodes infecting animals and humans from China. J Helminthol. 2012; in press.

19. Gasser RB, Zhu XQ, McManus DP. NADH dehydrogenase subunit 1 and cytochrome c oxidase subunit I sequences compared for members of the genus Taenia (Cestoda). Int J Parasitol. 1999; 29: 1965-1970.

20. Thompson JD, Gibson TJ, Plewniak F, Jeanmougin F, Higgins DG. The Clustal $\mathrm{X}$ windows interface: flexible strategies for multiple sequence alignment aided by quality analysis tools. Nucleic Acids Res. 1997; 24: 4876-4882.

21. Chilton NB, Gasser RB, Beveridge I. Differences in a ribosomal DNA sequence of morphologically indistinguishable species within the Hypodontus macropi complex (Nematoda: Strongyloidea). Int J Parasitol. 1995; 25: 647-651.

22. Lowe TM, Eddy SR. tRNAscan-SE: A program for improved detection of transfer RNA genes in genomic sequence. Nucleic Acids Res. 1997; 25: 955-964.

23. Librado P, Rozas J. DnaSP v5: a software for comprehensive analysis of DNA polymorphism data. Bioinformatics. 2009; 25: 1451-1452.

24. Tamura K, Peterson D, Peterson N, Stecher G, Nei M, Kumar S. MEGA5: molecular evolutionary genetics analysis using maximum likelihood, evolutionary distance, and maximum parsimony methods. Mol Biol Evol. 2011; 28: 2731-2739.

25. Talavera G, Castresana J. Improvement of phylogenies after removing divergent and ambiguously aligned blocks from protein sequence alignments. Syst Biol. 2007; 56: 564-577.

26. Ronquist F, Huelsenbeck JP. MrBayes 3: Bayesian phylogenetic inference under mixed models. Bioinformatics. 2003; 19: 1572-1574.

27. Guindon S, Gascuel O. A simple, fast, and accurate algorithm to estimate large phylogenies by maximum likelihood. Syst Biol. 2003; 52: 696-704.

28. Page RD. TREEVIEW: an application to display phylogenetic trees on personal. Comp Appl Biosci.1996; 12: 357-358.

29. Goddard JM, Wolstenholme DR. Origin and direction of replication in mitochondrial DNA molecules from the genus Drosophila. Nucleic Acids Res. 1980; 8: 741-757.

30. Nakao M, Yokoyama N, Sako Y Fukunaga M, Ito A. The complete mitochondrial DNA sequence of the cestode Echinococcus multilocularis (Cyclophyllidea: Taeniidae). Mitochondrion. 2002; 1: 497-509.

31. von Nickisch-Rosenegk M, Brown WM, Boore JL. Complete sequence of the mitochondrial genome of the tapeworm Hymenolepis diminuta: gene arrangements indicate that platyhelminths are eutrochozoans. Mol Biol Evol. 2001; 18: 721-730.

32. Nakao M, Sako Y, Ito A. The mitochondrial genome of the tapeworm Taenia solium: a finding of the abbreviated stop codon U. J Parasitol. 2003; 89: 633-635.

33. Jia WZ, Yan HB, Guo AJ, Zhu XQ, Wang YC, Shi WG, Chen HT, Zhan F, Zhang SH, Fu BQ, Littlewood DT, Cai XP. Complete mitochondrial genomes of Taenia multiceps, T. hydatigena and T. pisiformis: additional molecular markers for a tapeworm genus of human and animal health significance. BMC Genomics. 2010; 11: 447.

34. Nkouawa A, Sako Y, Nakao M, Nakaya K, Ito A. Loop-mediated isothermal amplification method for differentiation and rapid detection of Taenia species. J Clin Microbiol. 2009; 47: 168-174.

35. González LM, Montero E, Morakote N, Puente S, Díaz De Tuesta JL, Serra T, López-Velez R, McManus DP, Harrison LJ, Parkhouse RM, Gárate T. Differential diagnosis of Taenia saginata and Taenia saginata asiatica taeniasis through PCR. Diagn Microbiol Infect Dis. 2004; 49: 183-188.

36. Yamasaki H, Allan JC, Sato MO, Nakao M, Sako Y, Nakaya K, Qiu D, Mamuti W, Craig PS, Ito A. DNA differential diagnosis of taeni- 
asis and cysticercosis by multiplex PCR. J Clin Microbiol. 2004; 42: 548-553.

37. Okamoto M, Iseto C, Shibahara T, Sato MO, Wandra T, Craig PS, Ito A. Intraspecific variation of Spirometra erinaceieuropaei and phylogenetic relationship between Spirometra and Diphyllobothrium inferred from mitochondrial CO1 gene sequences. Parasitol Int. 2007; 56: 235-238.

38. Bray RA, Jones A, Andersen KI. Order Pseudophyllidea Carus, 1863. In: Khalil LF, Jones A, Bray RA, editors. Key to the Cestode Parasites of Vertebrates. Wallingford, UK: CBA International. 1994; 205-247. 